\title{
Unified Incremental Physical-Level and High-Level Synthesis
}

\author{
Zhenyu Gu, Student Member, IEEE, Jia Wang, \\ Robert P. Dick, Member, IEEE, and Hai Zhou, Senior Member, IEEE
}

\begin{abstract}
Achieving design closure is one of the biggest challenges for modern very large-scale integration system designers. This problem is exacerbated by the lack of high-level designautomation tools that consider the increasingly important impact of physical features, such as interconnect, on integrated circuit area, performance, and power consumption. Using physical information to guide decisions in the behavioral-level stage of system design is essential to solve this problem. In this paper, we present an incremental floorplanning high-level-synthesis system. This system integrates high-level and physical-design algorithms to concurrently improve a design's schedule, resource binding, and floorplan, thereby allowing the incremental exploration of the combined behavioral-level and physical-level design space. Compared with previous approaches that repeatedly call loosely coupled floorplanners for physical estimation, this approach has the benefits of efficiency, stability, and better quality of results. The average CPU time speedup resulting from unifying incremental physical-level and high-level synthesis is $24.72 \times$ and area improvement is $13.76 \%$. The low power consumption of a state-of-the-art low-power interconnect-aware high-levelsynthesis algorithm is maintained. The benefits of concurrent behavioral-level and physical-design optimization increased for larger problem instances.
\end{abstract}

Index Terms-Behavioral synthesis, floorplanning, low power design.

\section{INTRODUCTION}

$\mathbf{P}$ ROCESS SCALING has enabled the production of integrated circuits (ICs) with millions of transistors. This has allowed the design of more full-featured and high-performance ICs. However, these increased capabilities have come at a cost. In order to deal with increased design complexity and size, it is becoming increasingly important to automate higher levels of the design process.

High-level-synthesis systems [1]-[4] automatically convert behavioral algorithmic descriptions of design requirements, e.g., control data flow graphs (CDFGs) [5], into optimized register-transfer level (RTL) descriptions in languages such as VHDL or Verilog. Based on a behavioral description, a highlevel-synthesis system determines an allocation of resources,

Manuscript received November 24, 2005; revised September 22, 2006. This work was supported in part by the National Science Foundation under Awards CCR-0238484, CNS-0347941, and CNS-0613967, and in part by the Semiconductor Research Corporation under Award 2007-HJ-1593. This paper was recommended by Associate Editor A. Raghunathan.

The authors are with the Department of Electrical Engineering and Computer Science, Northwestern University, Evanston, IL 60208-3118 USA (e-mail: zgu646@eecs.northwestern.edu; jwa112@eecs.northwestern.edu; dickrp@ northwestern.edu; haizhou@eecs.northwestern.edu).

Digital Object Identifier 10.1109/TCAD.2007.895774 assignment of operations to resources, and a schedule for operations in an attempt to satisfy the design specifications and minimize some combination of delay, area, and power consumption [6]-[17]. Recently, in order to improve design area or performance estimation, researchers have considered the impact of physical details, e.g., floorplanning information, on high-level synthesis [18]-[23].

In the past, it was possible for high-level-synthesis algorithms to focus on logic, i.e., functional units (FUs) such as adders and multipliers. The contribution of wire delay and power was typically neglected without much loss of accuracy. Focusing on logic was once reasonable since logic was responsible for the majority of delay and power consumption. However, process scaling into the deep-submicrometer realm has changed the focus of very large-scale integration (VLSI) design from transistors to global interconnect. It is no longer possible to simplify the high-level-synthesis problem by ignoring interconnect.

Taking interconnect cost into consideration during highlevel synthesis has attracted significant attention. In previous work [24]-[28], the number of interconnects or multiplexers was used to estimate interconnect cost. The performance and power impacts of interconnect and interconnect buffers are now first-order timing and power considerations in VLSI design [29]. It is no longer possible to accurately predict the power consumption and performance of a design without first knowing enough about its floorplan to predict the structure of its interconnect. This change has dramatically complicated both design and synthesis. For this reason, a number of researchers have worked on interconnect-aware high-level-synthesis algorithms [30]-[32]. These approaches typically use a loosely coupled independent floorplanner for physical estimation. Although this technique improved on previous work by allowing estimation of physical properties, it has two drawbacks. First, the independent floorplanner may not be stable, i.e., a small change in the input netlist may result in a totally different floorplan. A move is a discrete change made to a solution during optimization that results in transition to a new position in the solution space. Floorplan instability may result in a high-level-synthesis algorithm that bases its moves on cost functions without continuity. Second, even if a floorplanner is stable, creating a floorplan from scratch for each high-level-synthesis move is inefficient, given the fact that the new floorplan frequently has only small differences with the previous one. The constructive approach works for small-problem instances but is unlikely to scale to large designs. New techniques for tightly coupling behavioral and physical synthesis that dramatically 
improve their combined performance and quality are now necessary.

Incremental automated design promises to build tighter relationship between high-level synthesis and physical design, improving the quality of each [33]-[35]. A number of high-level-synthesis algorithms are based on incremental optimization and are, therefore, amenable to integration with incremental physical-design algorithms. This has the potential of improving both quality and performance. Incremental methods improve quality of results by maintaining important physicallevel properties across consecutive physical estimations during synthesis. Moreover, they shorten CPU time by reusing and building upon previous high-quality physical-design solutions that required a huge amount of effort to produce.

This paper describes an incremental high-level-synthesis system that reduces synthesis time dramatically while producing ICs with better area and low power consumption compared to a state-of-the-art power-aware high-levelsynthesis algorithm. The benefits of this approach increase with increasing problem size and complexity. This paper is based on the interconnect-aware high-level-synthesis tool, ISCALP [31], which was based on the low-power data-path synthesis tool, SCALP [15]. We reuse the power modeling and iterativeimprovement high-level-synthesis framework from ISCALP. However, this paper differs from previous work in that a truly incremental floorplanner is used to estimate the interconnect structure [36]. In contrast, past work used a fast constructive algorithm. Moreover, the high-level-synthesis algorithm, itself, is made incremental. As shown in Section V, this resulted in an average speedup of $24.72 \times$ and an average area improvement of $13.76 \%$, while maintaining the low power consumption of a state-of-the-art power-aware high-level-synthesis algorithm. In addition, wire delay is considered in this paper to guarantee that the implementation meets its performance requirements.

This paper is organized as follows. Section II explains the motivation for this paper. Section III describes the design flow for the proposed high-level-synthesis system. The details of our incremental floorplanner are introduced in Section IV. Experimental results are presented in Section V. Conclusions and future work are presented in Section VI.

\section{Motivation}

In this section, we first present definitions useful in the discussion of high-level synthesis. Then, motivational examples are given based on our observations of the synthesis process. Examples are used to explain and motivate the use of unified high-level and physical-level optimization.

\section{A. Definitions and Background}

The input to ISCALP is a CDFG $G$, an input arrival (and output sampling) period $T_{s}$, and a library $L$ of FUs for data-path implementation. ISCALP produces an RTL circuit in which power consumption (including logic and wire power consumption) and estimated area are optimized. The ISCALP algorithm has two loops. Given the supply voltage, the outer loop incrementally reduces the number of control steps, csteps, from its maximum to minimum value, where csteps is defined as

$$
\text { csteps }=T_{s} \times f
$$

or alternatively,

$$
T_{\text {clock }}=T_{s} / \text { csteps } .
$$

In the above equations, each control step corresponds to a time period of one clock cycle, and the sample period $T_{s}$ is the constraint on the input data rate. The solution obtained from high-level synthesis must be able to process an input sample before the next one arrives. For a given specification, the sample period is fixed. Hence, csteps indicates the number of clock cycles required to process an input sample. The variable $f$ is the chip clock frequency. $T_{\text {clock }}$ is the chip clock period. ISCALP searches for the lowest power architecture for each possible value of csteps.

Given a value of csteps, which allows the clock period to be determined, the inner loop first uses the fastest available FU from the library to implement each operation. An as-soonas-possible (ASAP) schedule is then generated for the initial solution to determine whether it meets its timing requirements. The initial solution is then further optimized. Having obtained an initial solution that meets the sample period constraint for the current value of csteps, the iterative improvement phase attempts to improve the architecture by reducing the switched capacitance while satisfying the sample period constraints. More details can be found in literature [15], [31].

\section{B. Motivating Example}

ISCALP employs a fast constructive slicing floorplanner based on netlist partitioning and rotation/orientation selection to obtain a floorplan optimized for wire length and area [37], [38]. Although it improved on its predecessors by considering the impact of floorplanning on synthesis, there are several drawbacks to this approach.

First, an incremental high-level-synthesis algorithm only changes a small portion of the modules and connections in each move. However, a constructive (conventional) floorplanner always starts floorplanning from scratch. This is not efficient because the floorplan information obtained during the previous run is not reused. Moreover, it is possible that the newly produced floorplan will be totally different from the previous one, despite only small changes in the set of modules and interconnections. This lack of autocorrelation in floorplan solutions may result in a high-level-synthesis algorithm basing its future moves on information that immediately becomes invalid after the moves are applied.

Second, the efficiency of the constructive slicing-structure floorplanner decreases dramatically for blocks with nonunity aspect ratios (ISCALP assume blocks with unity aspect ratio). As a result of constraining the solution space to slicing floorplans, it is prone to reaching suboptimal solutions. However, simply replacing the slicing floorplanner with a high-quality floorplanner would result in unacceptably high CPU time. 
TABLE I

Numbers of MERges ANd CPU Times of DifFERENT BenChMARKS

\begin{tabular}{|c|c|c|c|c|c|c|c|c|c|c|c|c|}
\hline \multirow[b]{2}{*}{ Benchmark } & \multicolumn{6}{|c|}{ Unity aspect ratio } & \multicolumn{6}{|c|}{ Non-unity aspect ratio } \\
\hline & \multirow{2}{*}{\multicolumn{2}{|c|}{\begin{tabular}{|l|} 
No. of Merges \\
ISCALP IFP-HLS
\end{tabular}}} & \multirow{2}{*}{\begin{tabular}{|c|} 
No. of Splits \\
IFP-HLS
\end{tabular}} & \multirow{2}{*}{\multicolumn{3}{|c|}{$\begin{array}{c}\text { CPU Time } \\
\text { ISCALP (s) IFP-HLS (s) Speedup (x) }\end{array}$}} & \multirow{2}{*}{\multicolumn{2}{|c|}{$\begin{array}{c}\text { No. of Merges } \\
\text { ISCALP IFP-HLS }\end{array}$}} & \multirow{2}{*}{\begin{tabular}{|c|} 
No. of Splits \\
IFP-HLS
\end{tabular}} & \multirow{2}{*}{\multicolumn{3}{|c|}{$\begin{array}{c}\text { CPU Time } \\
\text { ISCALP (s) IFP-HLS (s) Speedup }(\times)\end{array}$}} \\
\hline CHEMICAL & & & & & & & & 1FF-1LD & & & & \\
\hline DCT_DIF & 981 & 492 & 1 & 102.98 & 120.86 & 0.85 & 769 & 508 & 1 & 699.96 & 102.27 & 6.84 \\
\hline DCT_IJPEG & 630 & 380 & 0 & 363.12 & 310.91 & 1.17 & 850 & 424 & 11 & 4297.13 & 183.36 & 23.44 \\
\hline DCT_LEE & 1512 & 674 & 2 & 248.07 & 194.41 & 1.28 & 1276 & 691 & 5 & 2669.18 & 166.56 & 16.03 \\
\hline DCT_WANG & 974 & 564 & 10 & 340.40 & 259.53 & 1.31 & 1019 & 515 & 3 & 5678.98 & 229.11 & 24.79 \\
\hline ELLIPTIC & 562 & 172 & 16 & 77.46 & 57.04 & 1.36 & 533 & 212 & 11 & 804.29 & 53.16 & 15.13 \\
\hline IIR77 & 858 & 506 & 2 & 214.89 & 192.87 & 1.11 & 858 & 426 & 1 & 2102.27 & 126.04 & 16.68 \\
\hline JACOBI_SM & 1652 & 572 & 52 & 1982.55 & 322.97 & 6.14 & 1755 & 530 & 26 & 31187.15 & 256.60 & 121.54 \\
\hline MAC & 220 & 31 & 13 & 7.64 & 10.28 & 0.74 & 200 & 19 & 14 & 22.12 & 5.64 & 3.92 \\
\hline PAULIN & 87 & 26 & 6 & 1.12 & 2.50 & 0.45 & 87 & 25 & 6 & 3.20 & 2.03 & 1.58 \\
\hline PR1 & 839 & 449 & 10 & 162.79 & 138.13 & 1.18 & 841 & 448 & 12 & 2041.15 & 121.94 & 16.74 \\
\hline PR2 & 1074 & 526 & 20 & 366.87 & 256.12 & 1.43 & 866 & 529 & 27 & 6145.86 & 177.36 & 34.65 \\
\hline SERIAL & 5200 & 2620 & 3 & 1187.37 & 767.48 & 1.55 & 5200 & 2660 & 1 & 14503.25 & 599.59 & 24.19 \\
\hline SMALL & 11 & 9 & 0 & 0.20 & 0.94 & 0.21 & 11 & 13 & 2 & 0.24 & 0.83 & 0.29 \\
\hline WDF & 1827 & 631 & 6 & 185.52 & 118.74 & 1.56 & 1588 & 739 & 9 & 1092.92 & 118.92 & 9.19 \\
\hline RANDOM100 & 1359 & 511 & 10 & 462.14 & 246.24 & 1.88 & 1353 & 433 & 5 & 5951.79 & 204.52 & 29.10 \\
\hline RANDOM 200 & 1110 & 780 & 1 & 16438.33 & 3498.53 & 4.70 & 1140 & 780 & 2 & 174540.95 & 2826.22 & 61.76 \\
\hline RANDOM300 & 2810 & 820 & 0 & 160997.92 & 18786.70 & 8.57 & $\mathrm{~N} / \mathrm{A}^{*}$ & 900 & 2 & N/A* & 12650.64 & N/A* \\
\hline Average & 1238.83 & 553.94 & 9.78 & 10179.04 & 1408.80 & 2.03 & $1113.59^{\prime}$ & $\begin{array}{l}537.76^{*} \\
\end{array}$ & 9.11 & $14854.96^{*}$ & $307.61^{*}$ & $24.72^{*}$ \\
\hline
\end{tabular}

*To solve non-unity aspect ratio Random300, ISCALP had not yet halted after 120 hours. The non-unity aspect ratio Random300 benchmark was excluded from the computation for average numbers.

TABLE II

CPU TIMES BREAK DOWN OF DiFFERENT BENCHMARKS

\begin{tabular}{|c|c|c|c|c|c|c|c|c|c|c|c|c|}
\hline \multirow{3}{*}{ Benchmark } & \multicolumn{6}{|c|}{ Unity aspect ratio } & \multicolumn{6}{|c|}{ Non-unity aspect ratio } \\
\hline & \multicolumn{3}{|c|}{ ISCALP } & \multicolumn{3}{|c|}{ IFP-HLS } & \multicolumn{3}{|c|}{ ISCALP } & \multicolumn{3}{|c|}{ IFP-HLS } \\
\hline & $\begin{array}{c}T_{f p} \\
\text { (s) }\end{array}$ & $\begin{array}{c}T_{\text {total }} \\
\text { (s) }\end{array}$ & $\begin{array}{c}\text { Ratio* } \\
(\%)\end{array}$ & $\begin{array}{c}T_{f p} \\
(\mathrm{~s})\end{array}$ & $\begin{array}{l}T_{\text {total }} \\
\text { (s) }\end{array}$ & $\begin{array}{c}\text { Ratio* } \\
(\%)\end{array}$ & $\begin{array}{c}T_{f p} \\
\text { (s) }\end{array}$ & $\begin{array}{c}T_{\text {total }} \\
\text { (s) }\end{array}$ & $\begin{array}{c}\text { Ratio* } \\
(\%)\end{array}$ & $\begin{array}{c}T_{f p} \\
(\mathrm{~s})\end{array}$ & $\begin{array}{c}T_{\text {total }} \\
\text { (s) }\end{array}$ & $\begin{array}{c}\text { Ratio* } \\
(\%)\end{array}$ \\
\hline CHEMICAL & 61.19 & 83.35 & 73.41 & 61.24 & 74.19 & 82.54 & 770.91 & 793.83 & 97.11 & 43.60 & 55.15 & 79.06 \\
\hline DCT_DIF & 75.57 & 102.98 & 73.38 & 101.86 & 120.86 & 84.28 & 674.93 & 699.96 & 96.42 & 82.95 & 102.27 & 81.11 \\
\hline DCT_IJPEG & 308.59 & 363.12 & 84.98 & 269.28 & 310.91 & 86.61 & 4238.01 & 4297.13 & 98.62 & 146.66 & 183.36 & 79.98 \\
\hline DCT_LEE & 191.11 & 248.07 & 77.04 & 159.66 & 194.41 & 82.13 & 2616.96 & 2669.18 & 98.04 & 130.88 & 166.56 & 78.58 \\
\hline DCT_WANG & 275.61 & 340.40 & 80.97 & 214.17 & 259.53 & 82.52 & 5613.21 & 5678.98 & 98.84 & 185.94 & 229.11 & 81.16 \\
\hline ELLIPTIC & 49.42 & 77.46 & 63.80 & 47.08 & 57.04 & 82.54 & 780.94 & 804.29 & 97.10 & 41.44 & 53.16 & 77.95 \\
\hline IIR77 & 168.91 & 214.89 & 78.60 & 164.32 & 192.87 & 85.20 & 2056.25 & 2102.27 & 97.81 & 104.88 & 126.04 & 83.21 \\
\hline JACOBI_SM & 1846.04 & 1982.55 & 93.11 & 245.88 & 322.97 & 76.13 & 31029.15 & 31187.15 & 99.49 & 188.11 & 256.60 & 73.31 \\
\hline MAC & 4.80 & 7.64 & 62.83 & 9.28 & 10.28 & 90.27 & 19.42 & 22.12 & 87.79 & 4.89 & 5.64 & 86.70 \\
\hline PAULIN & 0.54 & 1.12 & 48.21 & 2.11 & 2.50 & 84.40 & 2.50 & 3.20 & 78.13 & 1.66 & 2.03 & 81.77 \\
\hline PR1 & 126.84 & 162.79 & 77.92 & 112.32 & 138.13 & 81.31 & 2003.70 & 2041.15 & 98.17 & 96.38 & 121.94 & 79.04 \\
\hline PR2 & 299.53 & 366.87 & 81.64 & 213.68 & 256.12 & 83.43 & 6085.16 & 6145.86 & 99.01 & 141.40 & 177.36 & 79.72 \\
\hline SERIAL & 974.21 & 1187.37 & 82.05 & 634.26 & 767.48 & 82.64 & 14288.12 & 14503.25 & 98.52 & 476.38 & 599.59 & 79.45 \\
\hline SMALL & 0.09 & 0.20 & 45.00 & 0.84 & 0.94 & 89.36 & 0.15 & 0.24 & 62.50 & 0.71 & 0.83 & 85.54 \\
\hline WDF & 126.58 & 185.52 & 68.23 & 90.21 & 118.74 & 75.97 & 1037.36 & 1092.92 & 94.92 & 88.49 & 118.92 & 74.41 \\
\hline RANDOM100 & 383.64 & 462.14 & 83.01 & 208.30 & 246.24 & 84.59 & 5869.24 & 5951.79 & 98.61 & 172.87 & 204.52 & 84.52 \\
\hline RANDOM200 & 15484.14 & 16438.33 & 94.20 & 2877.49 & 3498.53 & 82.25 & 173591.19 & 174540.95 & 99.46 & 2220.85 & 2826.22 & 78.58 \\
\hline RANDOM300 & 151251.05 & 160997.92 & 93.95 & 15798.36 & 18786.70 & 84.09 & $\mathrm{~N} / \mathrm{A}^{* *}$ & $\mathrm{~N} / \mathrm{A}^{* *}$ & N/A** & 10089.21 & 12650.64 & 79.75 \\
\hline Average & 9534.88 & 10179.04 & 75.69 & 1178.35 & 1408.80 & 83.35 & $14745.72^{* *}$ & $14854.96^{* *}$ & $94.15^{* *}$ & $242.83^{* *}$ & $307.61^{* *}$ & $80.24^{* *}$ \\
\hline
\end{tabular}

${ }^{*}$ Ratio $=T_{f p} / T_{\text {total }}$

** To solve non-unity aspect ratio Random300, ISCALP had not yet halted after 120 hours. The non-unity aspect ratio Random300 benchmark was excluded from the computation for average numbers.

To solve these problems, we propose an incremental iterative improvement high-level-synthesis algorithm tightly integrated with a high-quality incremental floorplanner. This synthesis system is called incremental floorplanning high-level synthesis (IFP-HLS). We run the same benchmarks on both ISCALP and IFP-HLS, listing the number of merge operations and CPU time for each benchmark in Table I.

$$
t_{\text {total }}=N_{\text {moves }} *\left(t_{H L S}+t_{f p}\right)
$$

As (3) shows, the CPU time of the high-level-synthesis run can be divided into two parts: high-level-synthesis moves and the resulting physical design carried out by the floorplanner. As shown in Table II, floorplanning is the most time consuming of these. It uses at least $75.69 \%$ of the CPU time on average for both ISCALP and IFP-HLS. As shown in Table I, IFPHLS achieves an average reduction of 50\% in the number of high-level-synthesis merge operations compared to ISCALP. This results in a large reduction in floorplanner CPU time. The reduction in moves, and CPU time, is mainly due to the incremental high-level-synthesis and floorplanning algorithms used in IFP-HLS. Many high-level-synthesis moves result in time-consuming changes to the floorplan. IFP-HLS can greatly reduce CPU time by reducing the number of merge operations, particularly for larger benchmarks, which require larger solution spaces to be explored.

Fig. 1 illustrates the power consumptions of intermediate solutions during optimization in ISCALP and IFP-HLS. For 


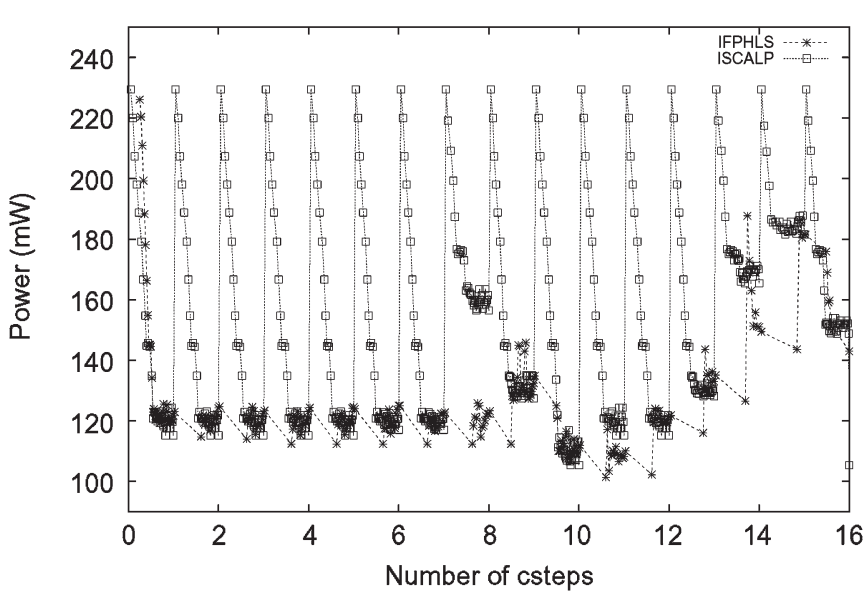

(a)

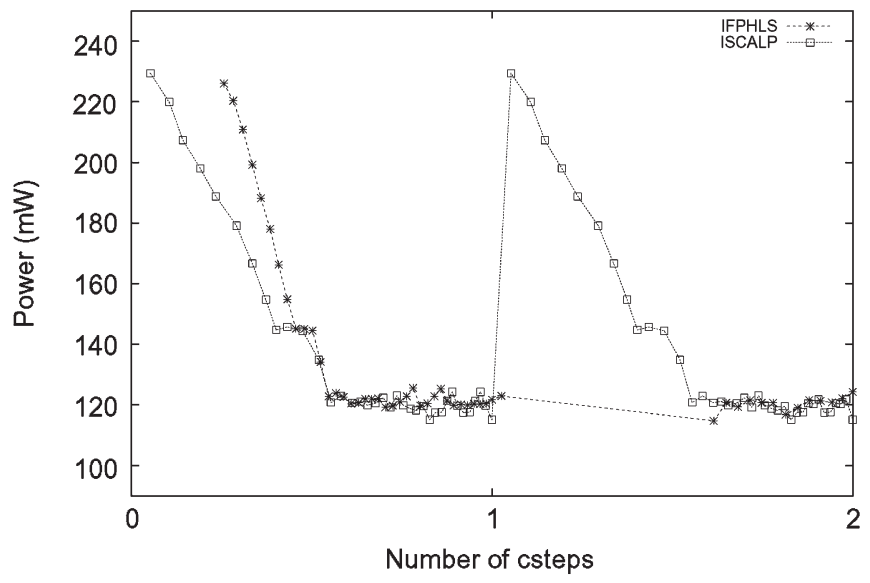

(b)

Fig. 1. Power consumption of intermediate solution during the optimization of ISCALP and IFP-HLS. (a) Power consumption of intermediate solution during the optimization for all the values of csteps. (b) Power consumption of intermediate solution during the optimization for the values of csteps from zero to two.

each value of csteps, we plot the intermediate solutions produced by the optimization algorithm. Note that all of these intermediate solutions have the same value of csteps. Incremental optimization allows IFP-HLS to focus on the most promising (low power) regions of the solution space while ISCALP must essentially restart optimization for each new potential clock frequency. This allows improvement to both optimization time and solution quality for IFP-HLS.

Note that ISCALP starts the floorplanner from scratch after each high-level design change. The incremental physical and architectural optimization used in IFP-HLS reduces CPU time dramatically, particularly for large applications. Table I indicates that the average CPU time speedup is $24.72 \times$. The improvement is greatest for the largest benchmarks. For example, when run on Random300, ISCALP does not finish within five days, while IFP-HLS finishes within $4 \mathrm{~h}$. In addition, IFP-HLS achieves $13.76 \%$ improvement in area compared to ISCALP.

The above examples clearly illustrate the value of using unified incremental physical-level and high-level synthesis. As shown in detail in Section $\mathrm{V}$, this approach improves both design quality and CPU time.

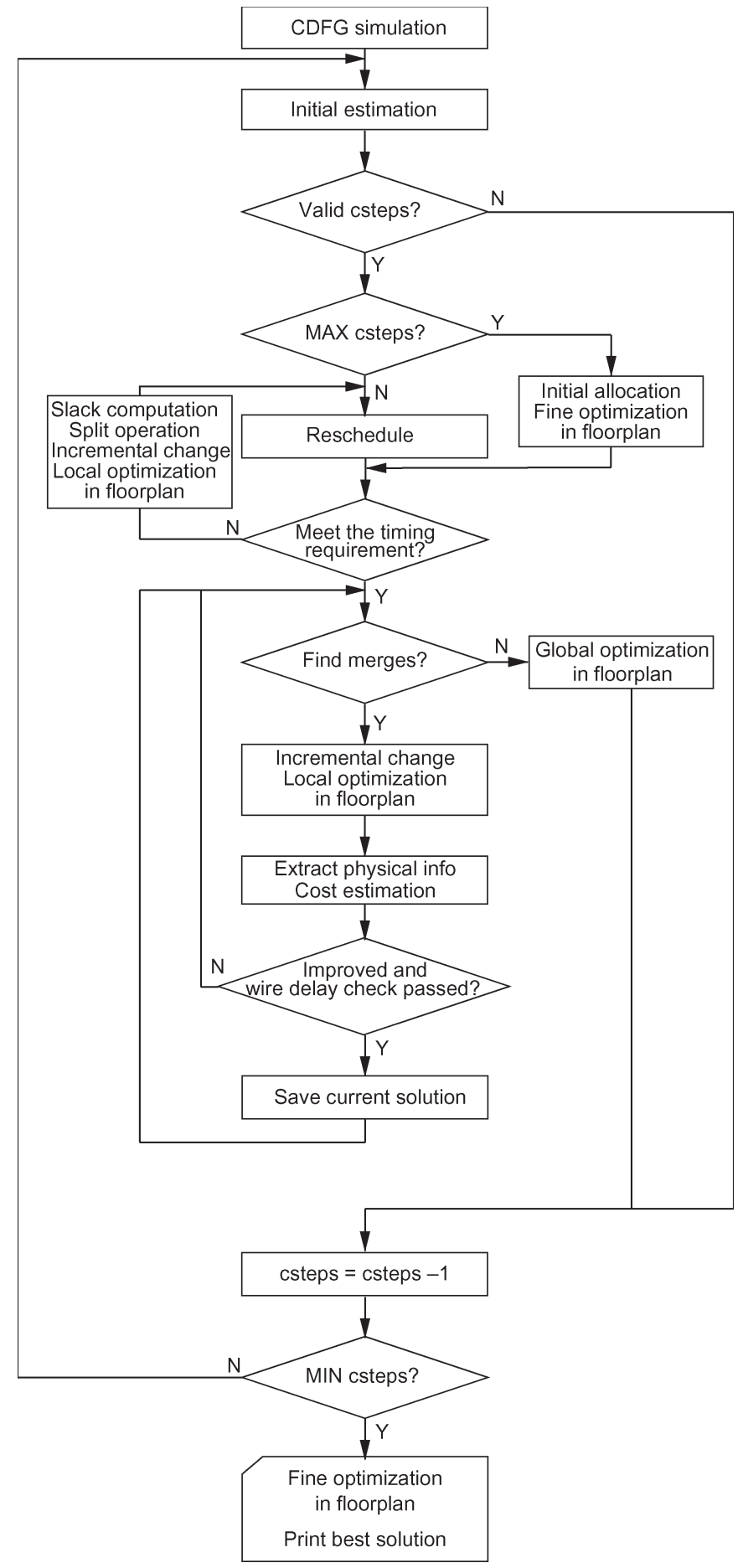

Fig. 2. Incremental high-level-synthesis algorithm.

\section{INCREMENTAL HIGH-LEVEL SYNTHESIS}

In this section, we describe IFP-HLS, the proposed incremental high-level synthesis algorithm. IFP-HLS is built upon ISCALP [31]. However, incorporating incremental floorplanning required substantial changes to that algorithm, resulting in a new low-power IFP-HLS algorithm. IFP-HLS considers both data-path and interconnect power consumption. As shown in Fig. 2, the CDFG is simulated with typical trace in order to profile the switching activity of each operation and data-transfer edge. An RTL design library is set up to provide the power and 
area information. The profiling information combined with this RTL design library and floorplanner is then used to evaluate the power consumption of both data path and interconnect. IFPHLS uses a new incremental method for improving FU binding during high-level synthesis. Although this improvement alone would result in a reduction in synthesis time, its motivation was to facilitate the integration of an incremental-floorplanning algorithm with high-level synthesis in order to improve solution quality and reduce synthesis time. This allows the highlevel-synthesis algorithm to determine the physical position of each module during optimization, enabling interconnect power consumption and delay estimation.

\section{A. Incremental High-Level-Synthesis Framework}

In this section, we give an overview of our incremental highlevel synthesis flow. The flowchart of IFP-HLS is shown in Fig. 2. IFP-HLS differs from ISCALP in a number of ways. Instead of generating an initial solution for each value of csteps, IFP-HLS only generates one solution at the maximum value of csteps and incrementally changes the solution as csteps decreases. Thus, in addition to using incremental floorplanning, IFP-HLS also eliminates redundant moves by taking advantages of incremental steps in high-level synthesis. Initially, we still use an ASAP schedule and fully parallel allocation to estimate whether there exists a valid solution for the current value of csteps. If not, it is not necessary to do further moves for the current number of control steps, because a binding that further reduces the finish time of an ASAP schedule is not possible. However, if an ASAP schedule that meets the timing requirements is possible, we will use the best solution from the previous value of csteps and reschedule it based on the current value, which is equal to the previous csteps minus one. If, after rescheduling, the solution meets its timing requirements, rebinding is not necessary. Otherwise, it will be necessary to parallelize some of the operations to improve performance. The split move is used to eliminate resource contention by splitting a pair of operations that were initially assigned to the same FU onto separate FUs. A detailed description of the split move may be found in Section III-B.

For a given value of csteps, when a move is chosen, IFP-HLS incrementally changes the floorplan to see whether the change improves solution quality. If so, the change is accepted. Otherwise, the change is rejected and other moves are attempted. This technique differs from that in ISCALP. In ISCALP, floorplanning is only done at the end of each csteps iteration; it does not take advantage of the solution correlation to save effort across csteps values. ISCALP uses only power consumption to guide the high-level-synthesis moves. In contrast, IFP-HLS uses a weighted sum of area and power consumption (in picowatts), with a ratio of $1 \mu \mathrm{m}^{2}$ to $5 \mathrm{pW}$, in order to evaluate solution quality.

A high-quality incremental floorplanner was developed and incorporated into IFP-HLS to guide the high-level-synthesis moves. Each time the high-level-synthesis algorithm needs physical information, it extracts that information from the current incrementally generated floorplan. Costs derived from the floorplan are also used to guide the high-level-synthesis moves.
By using incremental floorplanning, closer interaction between high-level synthesis and physical design is possible, i.e., the high-level-synthesis algorithm may determine the impact of potential changes to binding upon physical attributes such as interconnect power consumption and area.

The core idea of incremental design is to maintain good physical-level properties across consecutive physical estimations during the high-level-synthesis moves. It is possible to apply the idea of using an incremental optimization framework to integrate other algorithms, provided that the algorithms at each level of design can be made incremental. Let us consider a few other examples. In force-directed scheduling, all the operations may be scheduled iteratively in the order of increasing cost. The cost of scheduling each unscheduled operation is updated after each operation is scheduled. This provides a potential opportunity to tightly integrate an incremental floorplanner to allow physical-information feedback. Maximal clique is NPhard. Therefore, for maximal clique-based resource sharing, a heuristic is often used in practice. As long as the heuristic is iterative, it can be made incremental.

In summary, IFP-HLS performs scheduling, allocation, and binding by iteratively changing csteps and determining whether operations need to be rescheduled or rebound (split) in order to meet timing constraints. At each step, the floorplan is updated and reoptimized.

\section{B. Extended Move}

This section describes split moves, rescheduling, and a new graph technique to determine split locations.

We observed that when csteps decreases by one, each individual operation takes, at most, the same number of control steps as it did for the previous value of csteps. Given that csteps is no less than the previous csteps minus one, we can conclude that the ASAP schedule for the previous value of csteps violates the deadline for the current value of csteps by, at most, one clock cycle. We will use node $i$ 's slack $S_{i}$ to represent this information, which is defined as follows:

$$
S_{i}=L S T_{i}-E S T_{i}
$$

where $E S T_{i}$ is the earliest start time, and $L S T_{i}$ is the latest start time, which were computed by a topological sort. Hardwareresource contention has already been considered.

Nodes with non-negative slack values do not imply timing violations. However, nodes with slack values of -1 cause timing violations, i.e., they must be executed one cycle earlier. These timing violations can be removed by splitting merged operations which, although useful for previous values of csteps, now harm performance. Based on this observation, the split move is used to eliminate timing violations. Therefore, the whole highlevel-synthesis algorithm is implemented in an incremental way, from maximum to minimum values of csteps, without rebinding from scratch at each value of csteps. Few changes to binding and scheduling are required as a result of singleunit change to csteps. However, in order to meet timing requirements, it is sometimes necessary to split operators mapped to the same FU. The split move makes it possible to quickly apply these isolated changes. Previous high-level-synthesis 
systems, e.g., SCALP and ISCALP, started from a fully parallel implementation for each value of csteps and repeatedly merged operators to reduce area. Although both techniques are reasonable in the absence of an integrated floorplanner, the incremental approach used in IFP-HLS hastens optimization (without degrading solution quality) by requiring far fewer changes to the floorplan. Used together, the split and merge moves allow complete exploration of the solution space. However, the primary goal of changing the number of control steps is meeting timing constraints. We therefore start our exploration of the solution space at the most promising region by iteratively splitting FUs on the critical-timing path.

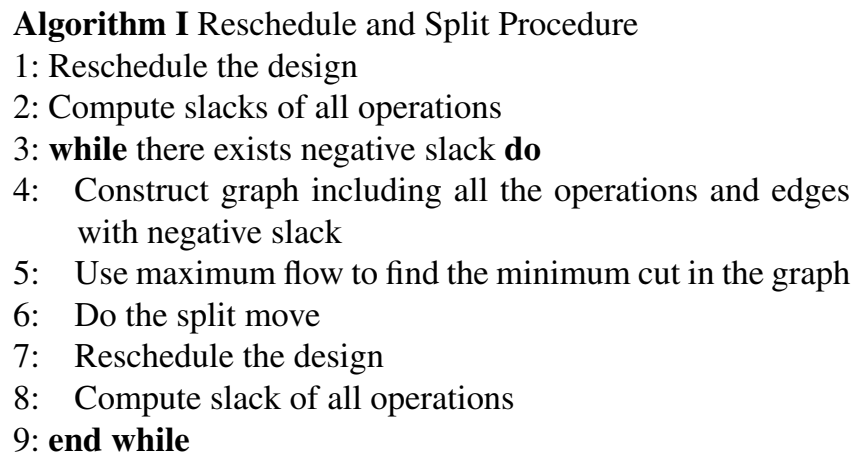

The reschedule and split procedure is shown in Algorithm 1. We will give an example to further describe this procedure. Consider the data flow graph shown in Fig. 3(a), in which arrows represent the data dependencies. Scheduling and allocation yield the DFG in Fig. 3(b). Here, we can see that three FUs are used. Operations ${ }^{*} 1$ and ${ }^{*} 2$ share FU1, operations ${ }^{*} 3,{ }^{*} 4$, and $* 5$ share FU2, and operation $+1,+2$, and +3 share FU3. The sample period is $108 \mathrm{~ns}$; each multiplication takes $20 \mathrm{~ns}$, and each addition takes $10 \mathrm{~ns}$. When csteps is reduced from ten to nine, instead of binding and scheduling from scratch, the algorithm reschedules based on current binding. For this example, after rescheduling, there are still timing violations for operations ${ }^{*} 3,{ }^{*} 4$, and ${ }^{*} 5$ because they were all bound to FU2, as shown in Fig. 3(c). Therefore, the split move is necessary in order to allow all operations to meet their timing requirements (a description of the split move follows).

Based on the result of slack computation, we produce a graph including all the operations with negative slack. Each operation is represented by a node. In addition, there are three kinds of edges, which are defined as follows.

1) Data-dependence edges, indicating that the destination node takes the source node's data as input.

2) Merge edges, indicating that the two nodes are currently bound to the same FU or same storage unit.

3) Pseudoedges, used to restructure the graph for application of the min-cut algorithm. Pseudosource and pseudosink nodes are introduced into the graph. All input nodes are connected to the pseudosource node and all output nodes are connected to the pseudosink node.

After constructing this graph, a min-cut algorithm is executed. First, an infinite capacity is assigned to all the pseudoedges and data-dependence edges. Merge edges are each

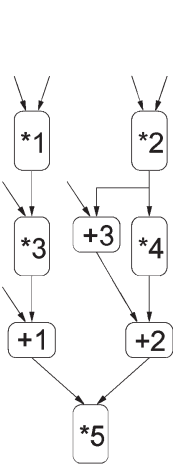

(a)

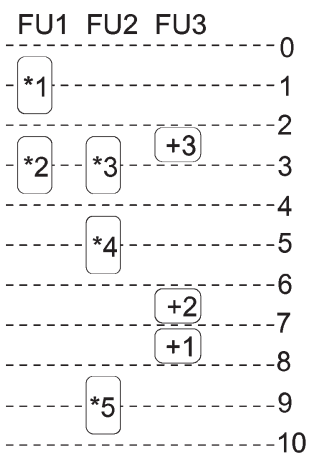

(b)

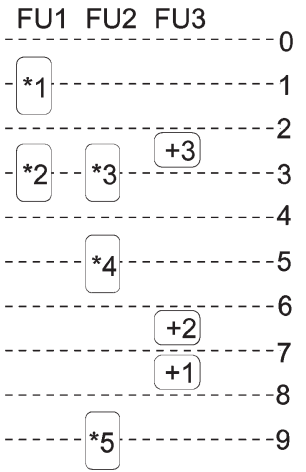

(c)

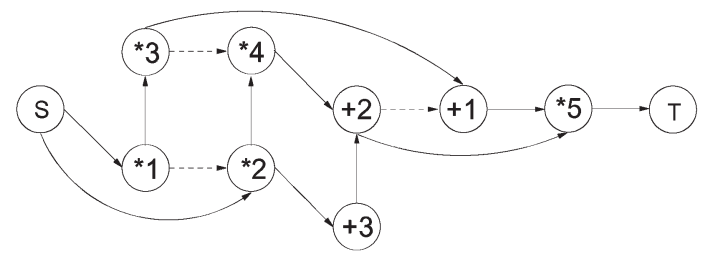

(d)

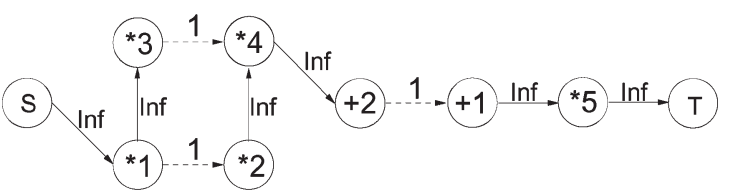

(e)

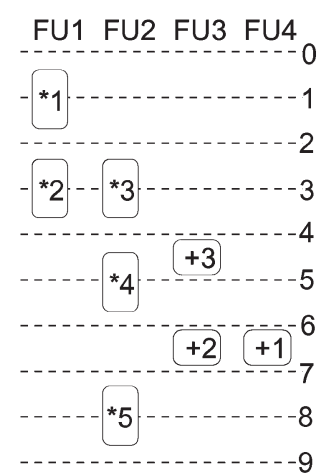

(f)

Fig. 3. Incremental changes in high-level synthesis. (a) CDFG. (b) Scheduling and allocation. (c) Timing violation. (d) Slack computation. (e) Min-cut flow. (f) After split move.

given capacities of one. If two nodes are connected by both the data-dependence edge and the merge edge, the merge edge is eliminated because split moves on nodes sharing dependence edges do not improve the timing properties. Using a min-cut algorithm in this manner splits a minimal cardinality subset of nodes, allowing a reduction in the finish time of the ASAP schedule. One could consider the impact of area and power consumption within the min-cut-max-flow algorithm by weighting the edges appropriately. However, this would generally lead to additional split operations, increasing CPU time.

Although decrementing csteps may increase delay by at most one clock cycle, there may be some value of csteps for which even fully parallel bindings do not allow an ASAP schedule to meet its timing constraints. Therefore, min-cut and rescheduling may not be carried out for some values of csteps. 


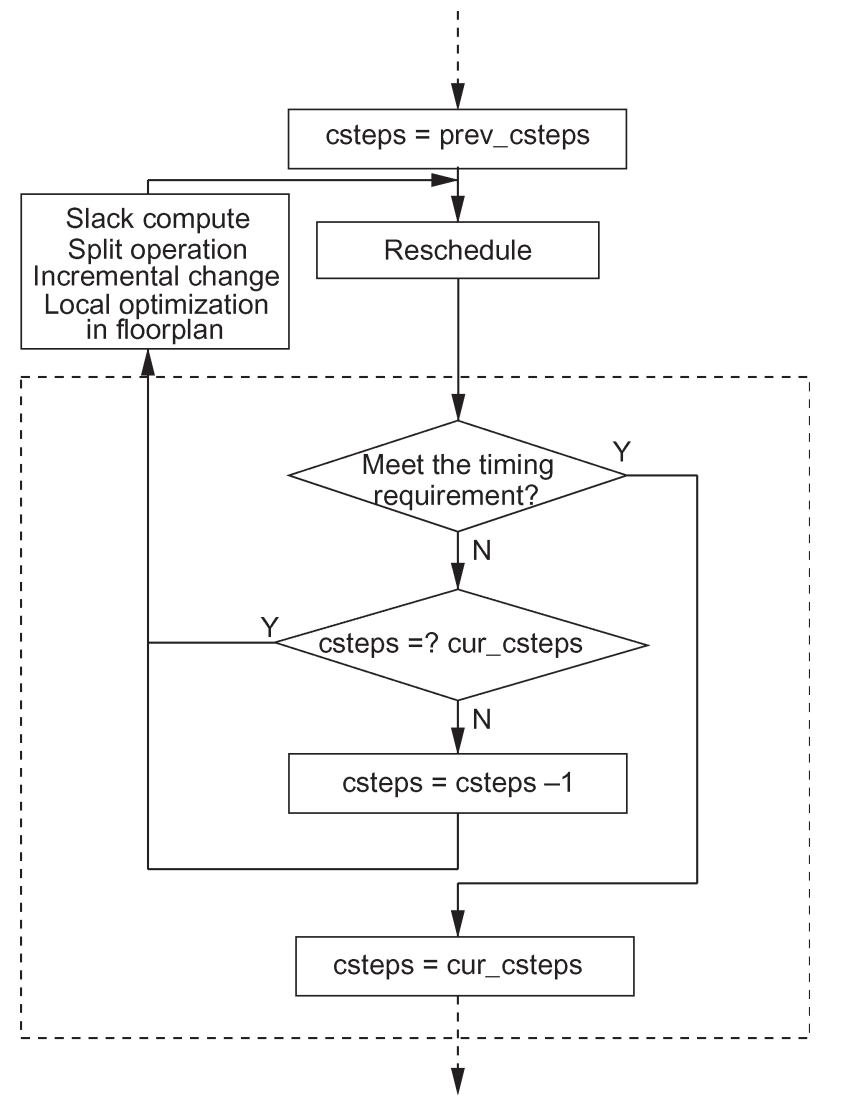

Fig. 4. Iterative split move for a slack smaller than -1 .

After the split move, the operations are rescheduled, and the slack is recomputed to determine whether timing constraints are met.

The algorithm described above was used to construct the graph shown in Fig. 3(d). The dashed lines represent merge edges. The solid lines represent pseudoedges and datadependence edges. Nodes $\mathrm{S}$ and $\mathrm{T}$ represent pseudosource and pseudosink nodes, respectively. After slack computation, we eliminate all the nodes and edges which are not on the critical path and assign a capacity of one to merge edges and a capacity of infinity to other edges, as shown in Fig. 3(e). For this example, it is possible to cut through either edge $(+2,+1)$, or edges $\left({ }^{*} 3,{ }^{*} 1\right)$ and $\left({ }^{*} 4,{ }^{*} 2\right)$. Here, we cut through +2 and +1 , which is the minimal cut, thereby assigning +1 to a new $\mathrm{FU}$, FU4. Edges +3 and +2 remain bound to the original FU, FU3. As shown in Fig. 3(f), no operation violates timing constraints.

Another case must also be considered. If no valid solutions exist for the current value of csteps, IFP-HLS will skip further optimization and decrement csteps. IFP-HLS may reach a valid value of csteps after repeatedly decrementing csteps. In this case, the slack values for some operations may be less than -1 . Hence, the value of csteps is decremented, and the split move, followed by rescheduling, is repeated until a valid solution is produced. This process is as shown in Fig. 4.

\section{INCREMENTAL FLOORPLANNING}

As discussed in previous sections, in order to introduce incremental combined behavioral and physical optimization

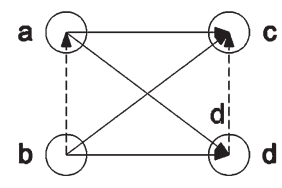

(a)

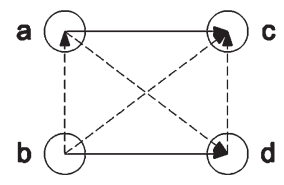

(b)
Fig. 5. (a) Horizontal cross and (b) vertical cross.

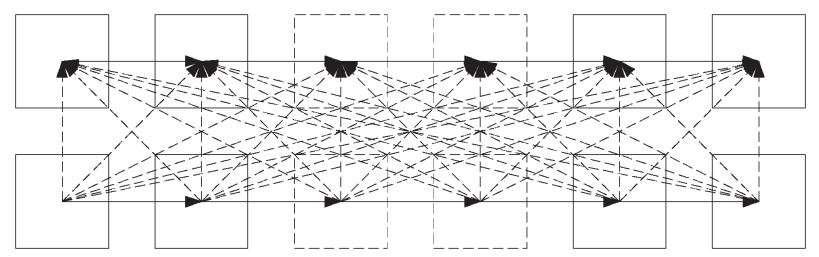

Fig. 6. Constraint graph without over-specifications and transitive edges can have quadratic number of edges.

into high-level synthesis, a high-quality incremental floorplanner is necessary. We have tested this idea by building an incremental simulated-annealing floorplanner into the IFPHLS algorithm. In this section, we describe this incremental floorplanner.

This floorplanner handles blocks with different aspect ratios and produces nonslicing floorplans. Unlike the netlistpartitioning approach used in ISCALP, it was designed primarily for quality, not speed. Although the impact on synthesis time would prevent the incorporation of a conventional high-quality floorplanner in the inner loop of a high-level-synthesis system, using incremental floorplanning enables both high-quality and low-synthesis time. High-level-synthesis moves typically remove a single module or split a module into two. Therefore, many changes are small, and their effects on the floorplan are mostly local. We reuse the modified previous floorplan as a starting point for each new floorplan. The previous floorplan is optimized. Therefore, reoptimization of the current floorplan to incorporate local changes is fast. In practice, we have found that this technique leads to quality-of-results and performance improvements over constructive floorplanning, even when compared with a very fast constructive floorplanner.

\section{A. Floorplan Representation}

The adjacent constraint graph (ACG) floorplan representation is used within IFP-HLS's incremental floorplanner [36], [39], [40]. This representation will be briefly summarized here.

An ACG is a constraint graph satisfying the following three conditions: first, there is, at most, one relation (either horizontal or vertical) between any pair of vertices; second, there are no transitive edges; and third, there are no crosses. A cross is a special edge configuration that can result in quadratic number of edges in the constraint graph. Fig. 5 shows two cases of crosses, and Fig. 6 shows that a constraint graph with crosses may have quadratic number of edges even when the first two conditions are met. It is proved that the number of edges in an ACG is, at most, $O\left(n^{1.5}\right)$, where $n$ is the number of vertices [40].

The operations of removing and inserting vertices in an existing ACG are designed to reflect high-level binding decisions, i.e., merging and splitting. To obtain the physical position 
of each module, packing that is based on the longest path computation is employed since the ACG itself is a constraint graph.

Perturbations on the graph are designed so that the ACG can be used in an iterative optimization heuristic such as simulated annealing. They change the graph topology locally and have straightforward meanings in physical space. Since the interconnect lengths are determined by the physical positions of modules, which in turn depend on the graph topology, applying these perturbations changes the interconnects locally. Other perturbations include rotating a module and exchanging the two modules represented by any pair of vertices. The latter changes the interconnect globally.

\section{B. Incremental Floorplanner}

There are four situations in which the incremental floorplanner is called by the IFP-HLS framework. First, a floorplan should be generated after each ASAP schedule is produced. We call this an initial floorplanning. Second, a floorplan should be modified and optimized after each high-level-synthesis move. We call this per-move floorplanning. Third, for each csteps value, a floorplan for the best binding should be generated and compared to the best existing floorplan. We call this percstep floorplanning. Fourth, after determining the best clock frequency and binding, floorplanning is carried out to provide the final result. We call this final floorplanning.

Although initial, per-cstep, and final floorplanning are done with simulated annealing for quality, per-move floorplanning requires fewer global changes and less hill climbing. Moreover, perturbations resulting from high temperatures may disrupt high-quality floorplan structures. Therefore, it is reasonable to use lower temperatures for per-move floorplanning. In practice, we have found that using a temperature of zero results in good quality and performance. In other words, although simulated annealing is necessary in many cases, per-move floorplanning is done with a greedy iterative-improvement algorithm.

The details of our approach follow. First, after generating the first ASAP schedule and binding, we have an initial set of modules and interconnections. Simulated annealing is used to obtain an initial floorplan. Since every interconnect net has exactly one driving module, multipin nets are broken into two-pin wires with the driving module as the source. The wire length is calculated as the Manhattan distance between the two modules connected by the wire. At this point, the unit-length switched capacitances of data transfers between two modules are available. We use these as weights for the wire lengths. The weighted total wire length is related to power consumption, i.e., optimizing the weighted wire length minimizes interconnect power consumption. A weighted sum of the area and the interconnect power consumption is calculated as the floorplanner's cost function, i.e.,

$$
A+w \sum_{e \in E} C_{e} D_{e}
$$

where $A$ is the area, $w$ is the power-consumption weight, $E$ is the set of all wires, $e$ is an interconnect wire, $C_{e}$ is the unit-length switched capacitance for the data transfer along $e$, and $D_{e}$ is the length of $e$. With this approach, we optimize the floorplan for both the interconnect power consumption and the area. The resulting floorplan will be improved during the consecutive IFP-HLS moves. Therefore, the number of simulated annealing iterations is bounded to reduce synthesis time.

After each high-level-synthesis move, per-move floorplanning first modifies the previous floorplan by removing or splitting a module. The modules and switched capacitances are updated based upon the impact of these merges and splits. The floorplan is then reoptimized with a greedy iterativeimprovement algorithm using the same cost function as the simulated annealing algorithm. The greedy improvements are divided into consecutive rounds. In every round, we apply the same number of perturbations to the floorplan. If less than $10 \%$ of the perturbations result in reduced costs, reoptimization stops. Although it would be easy to use a low simulated annealing temperature to allow some hill climbing during reoptimization, this was not necessary in practice. It should be pointed out that changes to switched capacitances may require a few global changes in the ACG to obtain power-consumption-optimized floorplans. Therefore, we still allow the exchange perturbation to change the floorplan globally but reduce its frequency to favor local perturbations.

When we find the best binding for a given value of csteps, we do per-cstep floorplanning and compare the result with the best floorplan from previous value of csteps. This time, nonzero temperature simulated annealing is used because it increases floorplan quality. These normal simulated annealing runs occur only once per csteps value, allowing their CPU costs to be amortized.

After determining the best binding across all the possible values of csteps, a final floorplanning run is carried out for that binding. This final floorplanning occurs only once per synthesis run. Therefore, it is acceptable to use a higher quality, but slower, annealing schedule than those in the inner loop of highlevel synthesis, thereby reducing chip area and interconnect power consumption.

During the annealing schedule, we use a constant multiplicative cooling factor $r$, i.e.,

$$
T^{\prime}=r \times T
$$

where $T$ is the current temperature, and $T^{\prime}$ is the temperature for the next iteration. The cooling factors for initial, per-cstep, and final floorplannings are $0.7,0.8$, and 0.9 , respectively. At one temperature, if less than $10 \%$ of the perturbations are accepted, the annealing process stops. The ratio between the numbers of the perturbations at one temperature for initial, per-cstep, and final floorplannings is $1: 1: 5$. The number of perturbations per round for per-move floorplanning is the same as that in the final floorplanning.

The interconnect power-consumption weight $w$ is automatically computed during synthesis for each floorplanning run to avoid the difficulty of determining a proper value for all the situations. Before each floorplanning, we calculate the areato-power-consumption ratio $w_{0}$ using the existing floorplan, 
which is either the previous floorplan for per-move, per-cstep, and final floorplannings or the starting floorplan for initial floorplanning. For initial, per-cstep, and final floorplannings, the weight $w$ is set to $0.5 \cdot w_{0}$ to balance the area and the interconnect power consumption. For per-move floorplanning, it is more important to quickly provide a prediction of the trend of interconnect power consumption, so $w$ is set to $2.5 \cdot w_{0}$ instead. Note that, in this stage, it is not the area cost but the prediction of the interconnect power consumption that is the major consideration. Therefore, the wire-length weight was set to be a large value compared to the area weight.

\section{EXPERIMENTAL RESULTS}

In this section, we present the results produced by the IFPHLS algorithm described in Sections III and IV when run on a number of benchmarks. The results generated by ISCALP and IFP-HLS are compared. As explained in Section III-A, both approaches optimize area and power consumption. The experiments were conducted on Linux workstations with dual 933-MHz Pentium III processors and $512 \mathrm{MB}$ of random access memory.

\section{A. Benchmarks}

We evaluated 17 high-level-synthesis benchmarks using a $0.18-\mu \mathrm{m}$ technology library. Chemical and IIR77 are infiniteimpulse-response (IIR) filters. DCT_IJPEG is the Independent JPEG Group's implementation of digital cosine transform (DCT) [41]. DCT_Wang is a DCT algorithm named after the inventor [42]. Both DCT algorithms work on $8 \times 8$ arrays of pixels. Elliptic, an elliptic-wave filter, comes from the NCSU CBL high-level-synthesis benchmark suite [43]. Jacobi is the Jacobi iterative algorithm for solving a fourth-order linear system [44]. WDF is a finite-impulse-response-wave digital filter. The largest benchmark is Jacobi with 24 multiplications, 8 divisions, 8 additions, and 16 subtractions. In addition, we generate five CDFGs using a pseudorandom graph generator [45]. Random 100 has 20 additions, 15 subtractions, and 19 multiplications. Random 200 has 39 additions, 44 subtractions, and 36 multiplications. Random 300 has 59 additions, 58 subtractions, and 72 multiplications.

IFP-HLS had better performance than ISCALP on these large randomized benchmarks. In order to determine whether the improved performance of IFP-HLS was the result of random graph structure or benchmark size, we generated two structured benchmarks, Small and Serial. Small is composed of five operations connected in parallel. Serial is composed of 45 operations connected in serial. As shown in Table I, IFP-HLS has better CPU time for the structured large benchmark Serial. This is consistent with the results for other large benchmarks.

The area of each benchmark described in this section was estimated using presynthesized FUs (e.g., adders, multipliers, etc.) based on NEC's $0.18-\mu \mathrm{m}$ process and the floorplanner from the high-level-synthesis tool. The logic power consumption of each benchmark was evaluated using power models from the presynthesized FU-level design library. A full- system switching-activity simulator was used during powerconsumption computation. Wire power consumption and wire delay were calculated based on the wire capacitances estimated using Cong's and Pan's technique [46] and the wire-length information from the floorplanner of the high-level-synthesis design tools. As described in Section III, both logic and wire delays were calculated to determine whether each design meets its timing requirements. However, since the wire delay estimation is only implemented in IFP-HLS, this function was not used when comparing to ISCALP.

\section{B. Results}

The results of running ISCALP and IFP-HLS on nonunity aspect ratio FUs are shown in Fig. 7. As shown in Fig. 7(a) and (b), and Table I, IFP-HLS achieves an average CPU-time speedup of $24.72 \times, 13.76 \%$ improvement in area, and 50\% reduction in the number of merge moves in comparison with ISCALP. Low power consumption is maintained.

ISCALP uses a constructive floorplanner that may suffer performance degradation when used with nonunity-aspect-ratio FUs. In order to determine whether the improvement in quality and run time were the result of the specific type of floorplanner used in ISCALP, we repeated all experiments using only unityaspect-ratio FUs. As shown in Fig. 8 and Tables I and III, the IFP-HLS algorithm achieves an average CPU-time speedup of $2.03 \times, 11.32 \%$ improvement in area, and $54 \%$ reduction in the number of merge moves, while maintaining the same low power consumption as ISCALP.

As shown in Figs. 7 and 8 and Tables I and III, IFP-HLS always has better CPU time than ISCALP for both nonunityand unity-aspect-ratio cases except for two very small unityaspect-ratio benchmarks (PAULIN and MAC). There are two contributors to CPU time [as shown in (3)]: the number of highlevel-synthesis moves and the resulting-floorplanning operations. ISCALP employs a fast constructive slicing floorplanner based on netlist-partitioning and rotation/orientation selection to obtain a floorplan optimized for wire length and area. It is faster than our simulated annealing floorplanner for small benchmarks with only a few blocks largely due to its determinism. The simulated annealing algorithm may revisit the same valid solutions multiple times before reaching the halting conditions, while the constructive-slicing floorplanner can quickly consider all slicing-structure floorplans, given small enough problem sizes. In contrast, the simulated annealing floorplanner is relatively faster on large-problem instances, because it can focus its moves on the most promising regions of the solution space, while the constructive floorplanner is left to explicitly consider an exponentially increasing number of points in the solution space. Please note that both floorplanners run quickly on small benchmarks. We are primarily concerned with floorplanner performance on large-problem instances, for which run-time is a concern. In addition, recall that ISCALP is an interconnect-aware power-driven high-level-synthesis tool. These results show that, on average, IFP-HLS achieves better CPU time and area while maintaining good power consumption. We also analyze the time break down between highlevel-synthesis moves and floorplanning. As shown in Table II, 


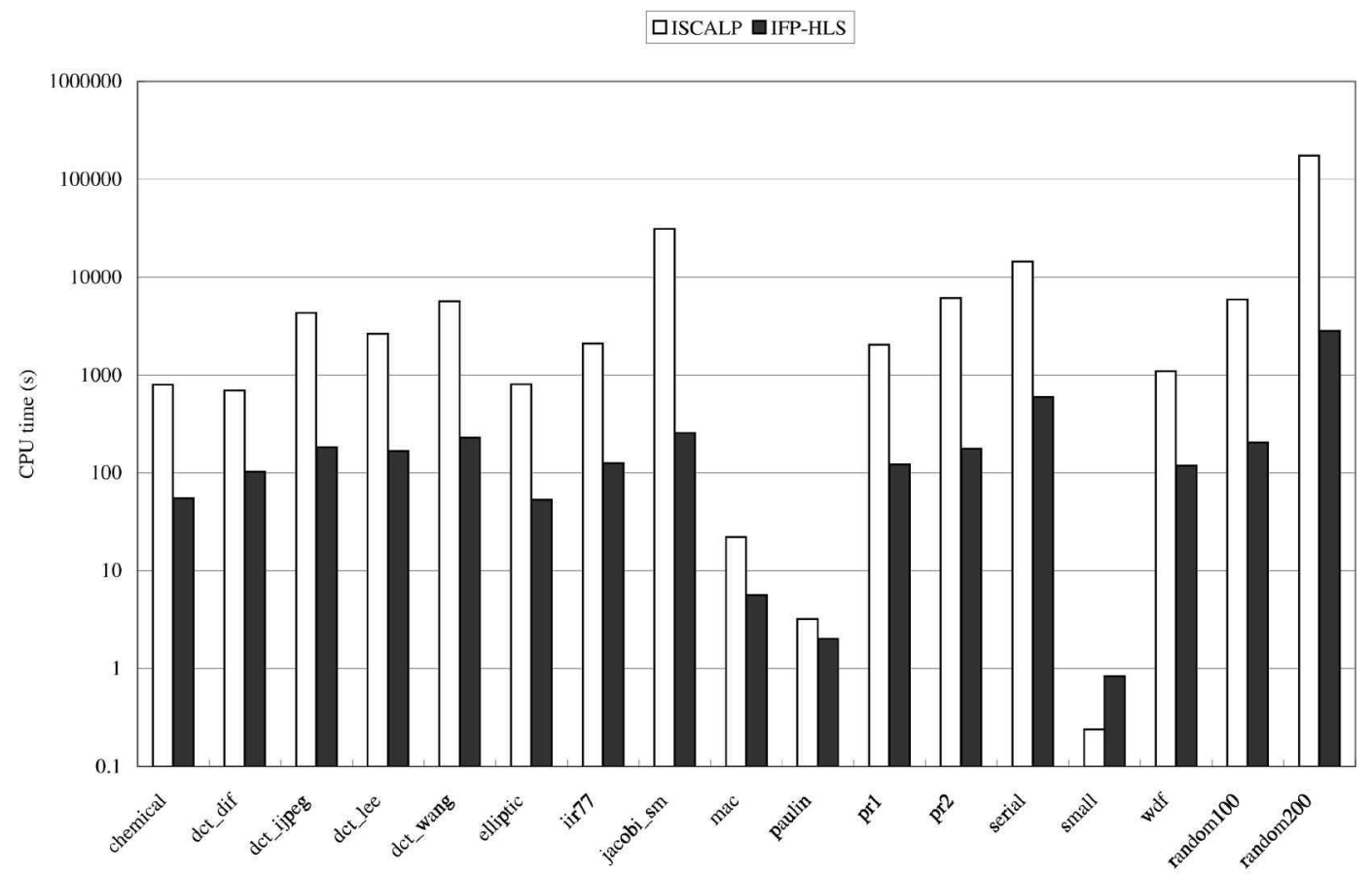

(a)

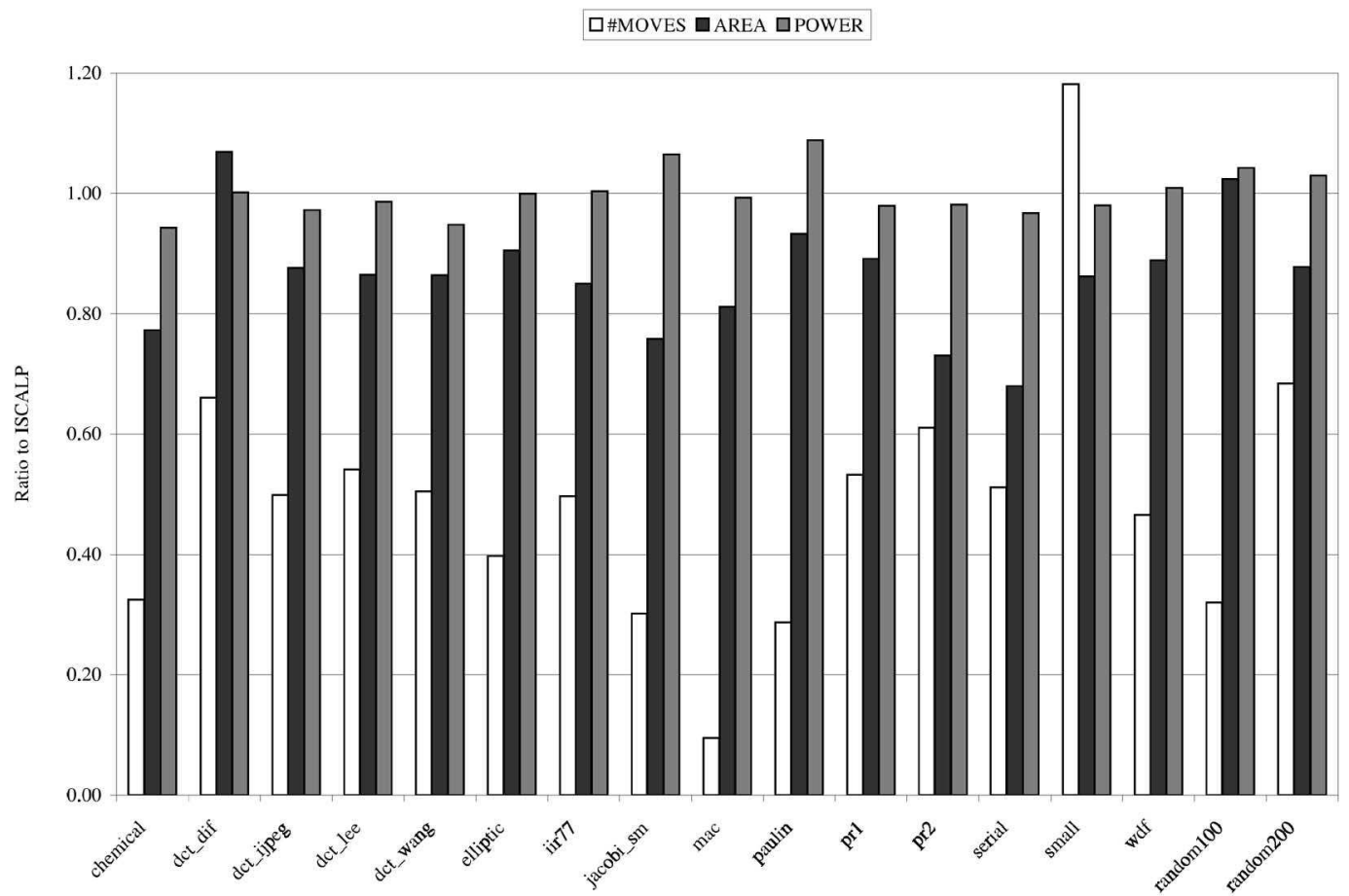

(b)

Fig. 7. Comparison between ISCALP and IFP-HLS for nonunity-aspect-ratio FUs. (a) CPU time for nonunity-aspect-ratio FUs. (b) Number of moves, area, and power consumption for nonunity-aspect-ratio FUs.

floorplanning used more than $75.69 \%$ of the total CPU time on average for both ISCALP and IFP-HLS; floorplanning is the most time-consuming part of the high-level-synthesis design flow.

In an attempt to isolate the impact of using a constructive floorplanner from the impact of using incremental optimization, we compared the results produced by running ISCALP, which is followed by a high-quality simulated annealing floorplanner by those produced by IFP-HLS. On average, this results in a $1.6 \%$ increase in area and $2.7 \%$ decrease in total power compared to IFP-HLS for unity-aspect-ratio FUs and a $0.8 \%$ increase in area and $1.3 \%$ decrease in total power consumption for nonunity-aspect-ratio FUs. Note that ISCALP aggressively optimizes power consumption. These results indicate that the incremental optimization algorithm within IFP-HLS permits comparable quality, using much less CPU time, as compared to 


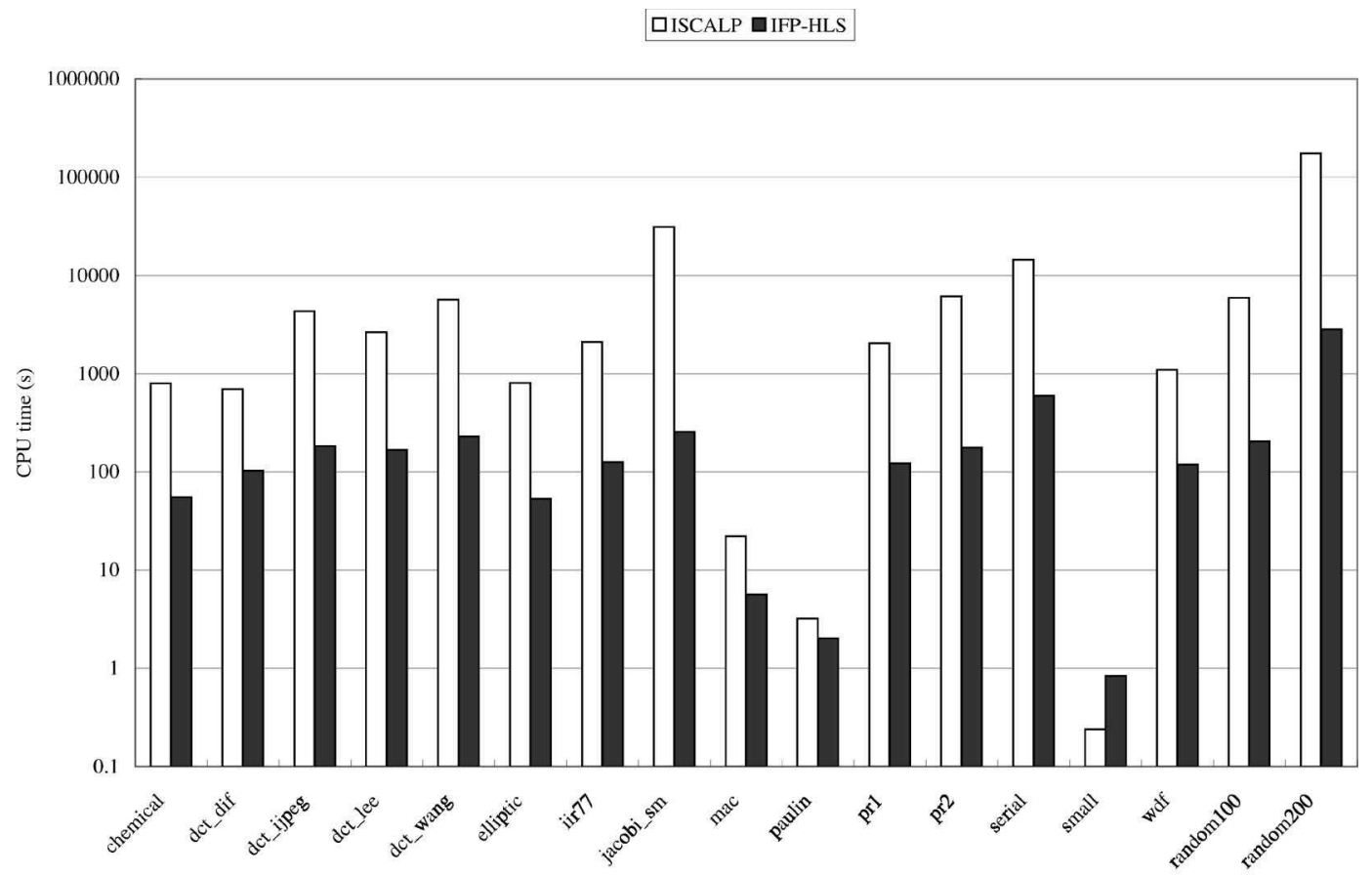

(a)

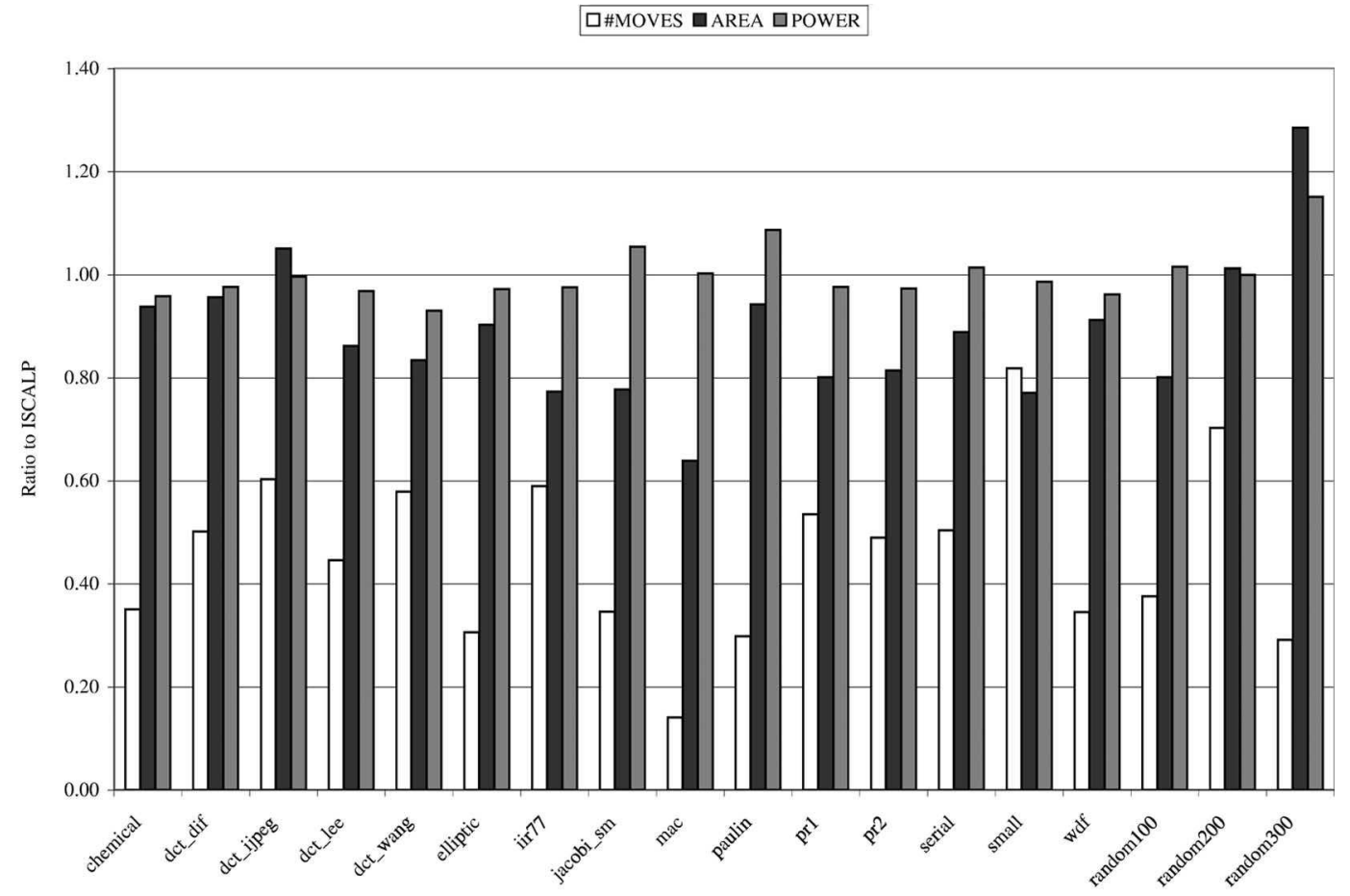

(b)

Fig. 8. Comparison between ISCALP and IFP-HLS for unity-aspect-ratio FUs. (a) CPU time for unity-aspect-ratio FUs. (b) Number of moves, area, and power consumption for unity-aspect-ratio FUs.

a nonincremental behavioral synthesis algorithm followed by an iterative improvement floorplanner.

\section{CONCLUSION}

This paper presented an IFP-HLS system that integrates high-level and physical-level design algorithms to concurrently improve a design's schedule, resource binding, and floorplan. Compared with previous approaches that repeatedly call loosely coupled floorplanners, this approach has the benefit of efficiency, stability, and better quality results. As shown in Section V, for nonunity-aspect ratio FUs, incremental floorplanning allowed an average CPU time speedup of $24.72 \times$ and an area improvement of $13.76 \%$. For unity-aspect-ratio FUs, 
TABLE III

AREA AND Power IMPRovemENTS OF DiFFERENT BENCHMARKS

\begin{tabular}{c|cc|cc|cc}
\hline \multirow{2}{*}{ Benchmark } & \multicolumn{2}{|c|}{ Area Improvement (\%) } & \multicolumn{2}{c|}{ Wire Power } & Improvement (\%) & \multicolumn{2}{c}{ Total Power Improvement (\%) } \\
& Unity & Non-unity & Unity & Non-unity & Unity & Non-unity \\
\hline CHEMICAL & 6.23 & 22.67 & 22.69 & 9.39 & 4.17 & 5.72 \\
DCT_DIF & 4.36 & -6.91 & 41.51 & 21.86 & 2.37 & -0.16 \\
DCT_IJPEG & -5.09 & 12.35 & 49.59 & -4.39 & 0.35 & 2.76 \\
DCT_LEE & 13.84 & 13.53 & 22.74 & 7.58 & 3.16 & 1.35 \\
DCT_WANG & 16.60 & 13.61 & 38.50 & 42.82 & 7.00 & 5.15 \\
ELLIPTIC & 9.70 & 9.45 & 22.18 & 28.32 & 2.77 & 0.03 \\
IIR77 & 22.69 & 15.03 & 21.32 & -0.37 & 2.42 & -0.34 \\
JACOBI_SM & 22.26 & 24.17 & 14.13 & 9.76 & -5.41 & -6.51 \\
MAC & 36.13 & 18.84 & 9.65 & 13.72 & -0.27 & 0.70 \\
PAULIN & 5.74 & 6.72 & 26.19 & 23.39 & -8.72 & -8.86 \\
PR1 & 19.86 & 10.87 & 6.13 & 16.44 & 2.39 & 2.05 \\
PR2 & 18.56 & 26.93 & 30.83 & -7.75 & 2.67 & 1.84 \\
SERIAL & 11.16 & 32.04 & 11.74 & 31.57 & -1.42 & 3.28 \\
SMALL & 22.89 & 13.78 & 36.93 & 48.83 & 1.36 & 1.98 \\
WDF & 8.79 & 11.10 & 9.87 & -12.50 & 3.79 & -0.91 \\
RANDOM100 & 19.88 & -2.41 & 14.67 & 19.94 & -1.55 & -4.24 \\
RANDOM200 & -1.22 & 12.20 & 19.45 & -1.90 & 0.01 & -3.01 \\
RANDOM300 & -28.55 & N/A* & 31.80 & N/A* & -15.11 & N/A* \\
\hline Average & 11.32 & $13.76^{*}$ & 23.89 & $14.51^{*}$ & 0.00 & $0.05^{*}$ \\
\hline
\end{tabular}

${ }^{*}$ To solve non-unity aspect ratio Random300, ISCALP had not yet halted after 120 hours. The non-unity aspect ratio Random 300 benchmark was excluded from the computation for average numbers.

the CPU-time speedup was $2.03 \times$ and area was improved by $11.32 \%$. In both cases, the low power consumption of a stateof-the-art low-power interconnect-aware high-level-synthesis algorithm was maintained. We conclude that incremental floorplanning improved the quality of synthesis results and improves performance dramatically, making synthesis from larger specifications practical.

\section{ACKNOWLEDGMENT}

The authors would like to thank Prof. N. Jha of Princeton University for the access to ISCALP and to NEC Labs America for the access to their $0.18-\mu \mathrm{m}$ technology library. They would also like to thank Y. Liu of Tsinghua University, Dr. A. Raghunathan of NEC Labs America, and Prof. L. Zhong of Rice University for their helpful suggestions.

\section{REFERENCES}

[1] R. Camposano and W. Wolf, High Level VLSI Synthesis. Norwell, MA: Kluwer, 1991.

[2] D. C. Ku and G. D. Micheli, High Level Synthesis of ASICs Under Timing and Synchronization Constraints. Norwell, MA: Kluwer, 1992.

[3] D. Gajski, N. Dutt, A. Wu, and S. Lin, High-Level Synthesis: Introduction to Chip and System Design. Norwell, MA: Kluwer, 1992.

[4] A. Raghunathan, N. K. Jha, and S. Dey, High-Level Power Analysis and Optimization. Norwell, MA: Kluwer, 1997.

[5] P. G. Paulin, J. P. Knight, and E. F. Girczyc, "HAL: A multi-paradigm approach to automatic data path synthesis," in Proc. Des. Autom. Conf., Jun. 1986, pp. 263-270.

[6] R. K. Gupta and G. De Micheli, "Hardware-software cosynthesis for digital systems," IEEE Des. Test Comput., vol. 10, no. 3, pp. 29-41, Sep. 1993.

[7] R. Mehra and J. Rabaey, "Behavioral level power estimation and exploration," in Proc. Int. Workshop Low Power Des., Apr. 1994, pp. 197-202.

[8] A. Dasgupta and R. Karri, "Simultaneous scheduling and binding for power minimization during microarchitecture synthesis," in Proc. Int. Symp. Low-Power Des., Apr. 1994, pp. 255-270.

[9] L. Goodby, A. Orailoglu, and P. M. Chau, "Microarchitecture synthesis of performance-constrained, low-power VLSI designs," in Proc. Int. Comput. Des., Oct. 1994, pp. 323-326.

[10] A. Raghunathan and N. K. Jha, "Behavioral synthesis for low power," in Proc. Int. Conf. Comput. Des., Oct. 1994, pp. 318-322.
[11] A. P. Chandrakasan, M. Potkonjak, R. Mehra, J. Rabaey, and R. Brodersen, "Optimizing power using transformations," IEEE Trans. Comput.-Aided Design Integr. Circuits Syst., vol. 14, no. 1, pp. 12-31, Jan. 1995.

[12] R. S. Martin and J. P. Knight, "Power profiler: Optimizing ASICs power consumption at the behavioral level," in Proc. Des. Autom. Conf., Jun. 1995, pp. 42-47.

[13] J. M. Chang and M. Pedram, "Register allocation and binding for low power," in Proc. Des. Autom. Conf., Jun. 1995, pp. 29-35.

[14] N. Kumar, S. Katkoori, L. Rader, and R. Vemuri, "Profile-driven behavioral synthesis for low-power VLSI systems," IEEE Des. Test Comput., vol. 12 , no. 3, pp. 70-84, 1995 .

[15] A. Raghunathan and N. K. Jha, "SCALP: An iterative-improvement-based low-power data path synthesis system," IEEE Trans. Comput.-Aided Design Integr. Circuits Syst., vol. 16, no. 11, pp. 1260-1277, Nov. 1997.

[16] K. S. Khouri, G. Lakshminarayana, and N. K. Jha, "High-level synthesis of low power control-flow intensive circuits," IEEE Trans. Comput.Aided Design Integr. Circuits Syst., vol. 18, no. 12, pp. 1715-1729, Dec. 1999.

[17] H. P. Peixoto and M. F. Jacome, "A new technique for estimating lower bounds on latency for high level synthesis," in Proc. Great Lakes Symp. VLSI, Mar. 2000, pp. 129-132.

[18] M. C. McFarland and T. J. Kowalski, "Incorporating bottom-up design into hardware synthesis," IEEE Trans. Comput.-Aided Design Integr. Circuits Syst., vol. 9, no. 9, pp. 938-950, Sep. 1990.

[19] D. W. Knapp, "Fasolt: A program for feedback-driven data-path optimization," IEEE Trans. Comput.-Aided Design Integr. Circuits Syst., vol. 11, no. 6, pp. 677-695, Jun. 1992.

[20] J. P. Weng and A. C. Parker, "3D scheduling: High-level synthesis with floorplanning," in Proc. Des. Autom. Conf., Jun. 1991, pp. 668-673.

[21] Y. M. Fang and D. F. Wong, "Simultaneous functional-unit binding and floorplanning," in Proc. Int. Conf. Comput.-Aided Des., Nov. 1994, pp. 317-321.

[22] M. Xu and F. J. Kurdahi, "Layout-driven RTL binding techniques for high-level synthesis using accurate estimators," ACM Trans. Des. Autom. Electron. Syst., vol. 2, no. 4, pp. 312-343, Oct. 1997.

[23] W. E. Dougherty and D. E. Thomas, "Unifying behavioral synthesis and physical design," in Proc. Des. Autom. Conf., Jun. 2000, pp. 756-761.

[24] P. G. Paulin and J. P. Knight, "Scheduling and binding algorithms for highlevel synthesis," in Proc. Des. Autom. Conf., Jun. 1989, pp. 1-6.

[25] C. A. Papachristou and H. Konuk, "A linear program driven scheduling and allocation method followed by an interconnect optimization algorithm," in Proc. Des. Autom. Conf., Jun. 1990, pp. 77-83.

[26] T. A. Ly, W. L. Elwood, and E. F. Girczyc, "A generalized interconnect model for data path synthesis," in Proc. Des. Autom. Conf., Jun. 1990, pp. $168-173$.

[27] S. Tarafdar and M. Leeser, "The DT-model: High-level synthesis using data transfer," in Proc. Des. Autom. Conf., Jun. 1998, pp. 114-117. 
[28] C. Jego, E. Casseau, and E. Martin, "Interconnect cost control during high-level synthesis," in Proc. Int. Conf. Des. Circuits Integr. Syst., Nov. 2000, pp. 507-512.

[29] R. Ho, K. Mai, and M. Horowitz, "The future of wires," Proc. IEEE, vol. 89, no. 4, pp. 490-504, Apr. 2001.

[30] P. Prabhakaran and P. Banerjee, "Simultaneous scheduling, binding and floorplanning in high-level synthesis," in Proc. Int. Conf. VLSI Des., Jan. 1998, pp. 428-434.

[31] L. Zhong and N. K. Jha, "Interconnect-aware low power high-level synthesis," IEEE Trans. Comput.-Aided Design Integr. Circuits Syst., vol. 24, no. 3, pp. 336-351, Mar. 2005.

[32] A. Stammermann, D. Helms, M. Schulte, A. Schulz, and W. Nebel, "Binding, allocation and floorplanning in low power high-level synthesis," in Proc. Int. Conf. Comput.-Aided Des., Nov. 2003, pp. 544-550.

[33] O. Coudert, J. Cong, S. Malik, and M. Sarrafzadeh, "Incremental CAD," in Proc. Int. Conf. Comput.-Aided Des., Nov. 2000, pp. 236-244.

[34] W. Choi and K. Bazargan, "Hierarchical global floorplacement using simulated annealing and network flow migration," in Proc. Des. Autom. Test Eur. Conf., Mar. 2003, pp. 1104-1105.

[35] Z. P. Gu, J. Wang, R. P. Dick, and H. Zhou, "Incremental exploration of the combined physical and behavioral design space," in Proc. Des. Autom. Conf., Jun. 2005, pp. 208-213.

[36] H. Zhou and J. Wang, "ACG-adjacent constraint graph for general floorplans," in Proc. Int. Comput. Des., Oct. 2004, pp. 572-575.

[37] C. M. Fiduccia and R. M. Mattheyses, "A linear-time heuristic for improving network partitions," in Proc. Des. Autom. Conf., Jun. 1982, pp. 173-181.

[38] L. Stockmeyer, "Optimal orientations of cells in slicing floorplan designs," Inf. Control, vol. 57, no. 2/3, pp. 91-101, May 1983.

[39] J. Wang and H. Zhou, "Interconnect estimation without packing via ACG floorplans," in Proc. Asia Pac. South Pac. Des. Autom. Conf., Jan. 2005, pp. $1152-1155$.

[40] J. Wang, "Floorplanning by adjacent constraint graph and its applications," M.S. thesis, Northwestern Univ., Evanston, IL, Jun. 2005

[41] Independent JPEG group. [Online]. Available: www.ijp.org

[42] K. R. Rao and P. Yip, Discrete Cosine Transform: Algorithms, Advantages, Applications. New York: Academic, 1990.

[43] NCSU CBL high-level synthesis benchmark suite. [Online]. Available: www.cbl.ncsu.edu/benchmarks

[44] S. Y. Kung, VLSI Array Processors. Englewood Cliffs, NJ: PrenticeHall, 1988.

[45] R. P. Dick, D. L. Rhodes, and W. Wolf, "TGFF: Task graphs for free," in Proc. Int. Workshop Hardware/Software Co-Des., Mar. 1998, pp. 97-101.

[46] J. Cong and Z. Pan, "Interconnect performance estimation models for design planning," IEEE Trans. Comput.-Aided Design Integr. Circuits Syst., vol. 20, no. 6, pp. 739-752, Jun. 2001.

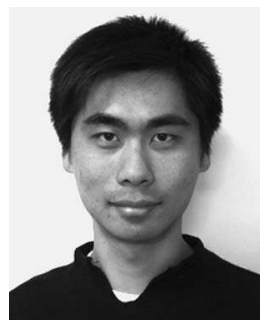

Zhenyu (Peter) Gu (S'04) received the B.S. and M.S. degrees from Fudan University, Shanghai, China, in 2000 and 2003, respectively. He is currently working toward the Ph.D. degree at the Department of Electrical Engineering and Computer Science, Northwestern University, Evanston, IL.

His research interests include thermal- and poweraware design automation of integrated circuits.

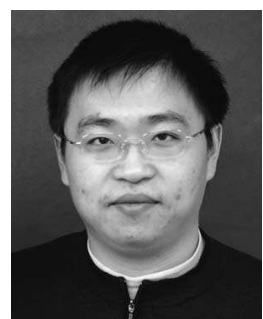

Jia Wang received the B.S. degree in electronic engineering from Tsinghua University, Beijing, China, in 2002, and the M.S. degree in computer engineering from Northwestern University, Evanston, IL, in 2005 . He is currently working toward the Ph.D. degree at the Department of Electrical Engineering and Computer Science, Northwestern University.

His research interests include computer-aided design of very large scale integrated circuits and algorithm design.

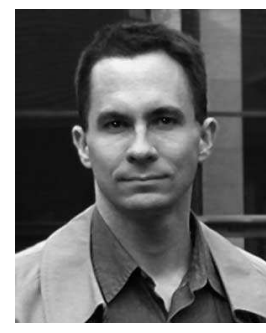

Robert P. Dick (S'95-M'02) received the B.S degree from Clarkson University, Potsdam, NY, and the Ph.D. degree from Princeton University, Princeton, NJ.

He worked as a Visiting Researcher at NEC Labs America and as a Visiting Professor at the Department of Electronic Engineering, Tsinghua University. He is currently an Assistant Professor at the Department of Electrical Engineering and Computer Science, Northwestern University, Evanston, IL. He has published in the areas of embedded system synthesis, embedded operating systems, low-power and temperature-aware integrated circuit design, data compression, reliability, behavioral synthesis, and mobile ad hoc network protocols.

Dr. Dick was the recipient of the NSF CAREER award and won his department's Best Teacher of the Year award in 2004. He is an Associate Editor of the IEEE TRANSACTIONS ON VERY LARGE SCALE INTEGRATION (VLSI) SYSTEMS and serves on the Technical Program Committees of several conferences.

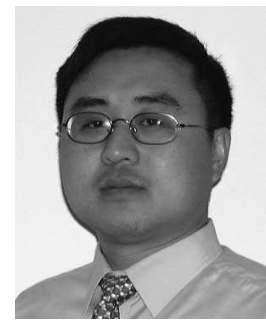

Hai Zhou (M'04-SM'04) received the B.S. and M.S. degrees in computer science and technology from Tsinghua University, Bejing, China, in 1992 and 1994, respectively, and the Ph.D. degree in computer sciences from The University of Texas at Austin, in 1999.

Before he joined the faculty of Northwestern University, Evanston, IL, he was with the Advanced Technology Group, Synopsys, Inc., Mountain View, CA. He is currently an Associate Professor of electrical engineering and computer science at Northwestern University. His research interests include very large scale integration computer-aided design, algorithm design, and formal methods.

Dr. Zhou served on the technical program committees of many conferences on very large-scale integrated circuits and computer-aided design. He was a recipient of the CAREER Award from the National Science Foundation in 2003. 